\title{
Bell Measurements and Observables
}

\author{
G. M. D'Ariano, P. Lo Presti and M. F. Sacchi \\ Theoretical Quantum Optics Group \\ Università degli Studi di Pavia and INFM Unità di Pavia \\ via A. Bassi 6, I-27100 Pavia, Italy
}

\begin{abstract}
A general matrix approach to study entangled states is presented, based on operator completeness relations. Bases of unitary operators are considered, with focus on irreducible representations of groups. Bell measurements for teleportation are considered, and robustness of teleportation to various kinds of non idealities is shown.
\end{abstract}

\section{Introduction}

Quantum mechanics builds up systems from subsystems in a fascinating way, through the tensor product, that allows one to set up the so called entangled states. These are states of the whole system that do not correspond to any state of the subsystems taken separately. This peculiar aspect of quantum world stands at the foundations of all the recent developments of quantum information theory, such as dense coding, teleportation, quantum computation, quantum cryptography, and so on [1]. These theoretical results have recently entered the realm of experimental physics [2].

Analogously to what happens for states, also quantum measurements on composite systems can be entangled when they are non local, namely they cannot be considered as a measurement jointly performed on the subsystems. In the general framework of positive operator valued measures (POVM), entangled measurements correspond to non factorizable POVM's. The so called "Bell measurements" are the most relevant example [3], corresponding to maximally entangled POVM's. Entanglement and Bell measurements are the basic ingredients of quantum teleportation.

In this letter, we present a matrix approach to address bipartite-system pure states along with general operator-completeness relations. These allow us to write the most general Bell-like POVM in compact form. Bases of unitary operators are considered, with focus on irreducible representation of groups. 
The canonical role of the groups $\mathbb{Z}_{N} \times \mathbb{Z}_{N}$ and Weyl-Heisenberg is analyzed. We conclude with a study of robustness of teleportation to different kinds of non idealities.

\section{Operator "basis"}

Consider a set of linear operators $\{B(\lambda), \lambda \in \Sigma, \Sigma$ Borel space $\}$ on a finite dimensional Hilbert space $\mathcal{H}$. This set is a "spanning set" for the operator space if it satisfies one of the following equivalent statements:

1. Completeness relation:

$$
\begin{aligned}
& \operatorname{Tr}\left[B^{\dagger}(\lambda) B\left(\lambda^{\prime}\right)\right]=\Delta\left(\lambda, \lambda^{\prime}\right), \quad \text { where } \int_{\Sigma} d \lambda \Delta\left(\lambda, \lambda^{\prime}\right) B(\lambda)=B\left(\lambda^{\prime}\right), \\
& \text { and } \quad \operatorname{Tr}\left[B^{\dagger}(\lambda) A\right]=0 \quad \forall \lambda \quad \Leftrightarrow \quad A=0 .
\end{aligned}
$$

2. For any linear operator $A$ on $\mathcal{H}$,

$$
\int_{\Sigma} d \lambda \operatorname{Tr}\left[B^{\dagger}(\lambda) A\right] B(\lambda)=A .
$$

3. Chosen any orthonormal basis $\{|i\rangle\}$ for $\mathcal{H}$,

$$
\int_{\Sigma} d \lambda\left\langle n\left|B^{\dagger}(\lambda)\right| m\right\rangle\langle l|B(\lambda)| k\rangle=\delta_{n k} \delta_{m l}
$$

4. For any linear operator $A$ on $\mathcal{H}$,

$$
\int_{\Sigma} d \lambda B^{\dagger}(\lambda) A B(\lambda)=\operatorname{Tr}[A] \mathbb{1}
$$

Proof of $1 \Leftrightarrow 2$ :

To prove $(\Rightarrow)$ we define $O=\int \operatorname{Tr}\left[B^{\dagger}(\lambda) A\right] B(\lambda) d \lambda-A$. Then we evaluate the following trace

$$
\operatorname{Tr}\left[B^{\dagger}\left(\lambda^{\prime}\right) O\right]=\int \operatorname{Tr}\left[B^{\dagger}\left(\lambda^{\prime}\right) B(\lambda)\right] \operatorname{Tr}\left[B^{\dagger}(\lambda) A\right] d \lambda-\operatorname{Tr}\left[B^{\dagger}\left(\lambda^{\prime}\right) A\right]=0,
$$

where integration has been carried out by means of the first line of Eq. (1); the second line of Eq. (1) completes the proof. Converse implication: the first line of Eq. (1) follows immediately by replacing $A$ with $B\left(\lambda^{\prime}\right)$ in Eq. (2), whereas the second part is a direct consequence of Eq. (2).

Proof of $2 \Leftrightarrow 3$ :

The proof of $(\Rightarrow)$ is immediate by substituting $A$ with $|m\rangle\langle n|$ in Eq. (2) and 
taking the matrix element between $\langle l|$ and $|k\rangle$. The converse is also straightforward: multiply both members of Eq. (3) by $\langle m|A| n\rangle|l\rangle\langle k|$ and take the sum over all indices $k, l, m, n$.

Proof of $3 \Leftrightarrow 4$ :

The direct implication is derived multiplying both members of Eq. (3) by $\langle m|A| l\rangle|n\rangle\langle k|$, and summing the result over all the indices $k, l, m, n$. To prove the converse, let $A=|m\rangle\langle l|$ in Eq. (4) and take the matrix element between $\langle n|$ and $|k\rangle$.

Note that Eq. (2) is exactly the linear decomposition of the operator $A$ on a set of operators $\{B(\lambda)\}$ induced by the scalar product $(B, A)=\operatorname{Tr}\left[B^{\dagger} A\right]$. For infinite dimensional Hilbert spaces the previous relations have meaning for Hilbert-Schmidt operators. However, they still hold for all linear operators in a distribution sense.

\section{General representation of bipartite-system pure states}

Chosen two orthonormal bases $\left\{|i\rangle_{1}\right\}$ and $\left\{|j\rangle_{2}\right\}$ for the Hilbert spaces $\mathcal{H}_{1}$ and $\mathcal{H}_{2}$ respectively, any vector $\left.|\psi\rangle\right\rangle \in \mathcal{H}_{1} \otimes \mathcal{H}_{2}$ can be written as

$$
\left.|\psi\rangle\rangle=\sum_{i j} c_{i j}|i\rangle_{1}|j\rangle_{2} \doteq|C\rangle\right\rangle
$$

Eq. (6) introduces a notation that exploits the correspondence between vectors in $\mathcal{H}_{1} \otimes \mathcal{H}_{2}$ and $N \times M$ matrices, where $\mathrm{N}$ and $\mathrm{M}$ are the dimensions of $\mathcal{H}_{1}$ and $\mathcal{H}_{2}$, respectively (cfr. Ref. [4]).

The following relations are an immediate consequence of Eq. (6)

$$
\begin{gathered}
\left.A \otimes B|C\rangle\rangle=\left|A C B^{T}\right\rangle\right\rangle, \quad\langle\langle A \mid B\rangle\rangle=\operatorname{Tr}\left[A^{\dagger} B\right], \\
\operatorname{Tr}_{2}[|A\rangle\rangle_{1212}\langle\langle B|]=\left(A B^{\dagger}\right)^{(1)}, \\
\operatorname{Tr}_{1}[|A\rangle\rangle_{1212}\langle\langle B|]=\left(A^{T} B^{*}\right)^{(2)} .
\end{gathered}
$$

Notice that the definition of the matrix $C$ in Eq. (6) is base-dependent, hence the transposition and conjugation in Eqs. (7) are referred to the same fixed basis. These relations are very useful for derivations and to express the results in an index-free compact form.

In the following we will focus our attention on bipartite systems whose Hilbert space is $\mathcal{H} \otimes \mathcal{H}$, with $N=\operatorname{dim}(\mathcal{H})$. As an application of the formalism just introduced, we give a direct proof of the existence of the Schmidt decomposition for a pure state of a bipartite system. Using a polar decomposition 
$A=V \sqrt{A^{\dagger} A}$, with $V$ unitary, which holds for any matrix $A$ [5], we choose a unitary operator $U$ so that $U A^{\dagger} A U^{\dagger}$ is diagonal, then we can write

$$
\left.\left.|A\rangle\rangle=\left|V \sqrt{A^{\dagger} A}\right\rangle\right\rangle=V U^{\dagger} \otimes U^{T}\left|U \sqrt{A^{\dagger} A} U^{\dagger}\right\rangle\right\rangle=\sum_{i} \sqrt{\lambda_{i}}|i\rangle_{1}^{\prime}|i\rangle_{2}^{\prime \prime},
$$

where $|i\rangle_{1}^{\prime}=V U^{\dagger}|i\rangle_{1},|i\rangle_{2}^{\prime \prime}=U^{T}|i\rangle_{2}$ and $\lambda_{i}$ is the eigenvalue of $A^{\dagger} A$ with eigenvector $|i\rangle$.

Using Eq. (6), it is straightforward to characterize maximally entangled states. These are defined as the states $|A\rangle\rangle$ whose partial trace on each of the two subsystems is proportional to identity; namely

$$
\operatorname{Tr}_{1}[|A\rangle\rangle\langle\langle A|]=A^{T} A^{*}=\frac{1}{N} \mathbb{1} \quad \text { and } \quad \operatorname{Tr}_{2}[|A\rangle\rangle\langle\langle A|]=A A^{\dagger}=\frac{1}{N} \mathbb{1},
$$

hence maximally entangled states are of the form

$$
\left.|A\rangle\rangle=\frac{1}{\sqrt{N}}|U\rangle\right\rangle
$$

with $U$ unitary. Two maximally entangled states are always connected by means of a local unitary transformation. In fact

$$
\left.|U\rangle\rangle=U V^{\dagger} \otimes \mathbb{1}|V\rangle\right\rangle
$$

Given a spanning set $\{B(\lambda)\}$, the set of vectors $\{|B(\lambda)\rangle\rangle\}$ spans $\mathcal{H} \otimes \mathcal{H}$ in the sense that

$$
\left.|A\rangle\rangle=\int d \lambda \operatorname{Tr}\left[B^{\dagger}(\lambda) A\right]|B(\lambda)\rangle\right\rangle
$$

Moreover one has

$$
\begin{aligned}
\left.\int d \lambda|B(\lambda)\rangle\right\rangle\langle\langle B(\lambda)| & \left.=\int d \lambda B(\lambda) \otimes \mathbb{1}|\mathbb{1}\rangle\right\rangle\left\langle\langle\mathbb{1}| B^{\dagger}(\lambda) \otimes \mathbb{1}=\right. \\
& =\operatorname{Tr}_{1}[|\mathbb{1}\rangle\rangle\langle\langle\mathbb{1}|]=\mathbb{1},
\end{aligned}
$$

hence the projectors on $|B(\lambda)\rangle\rangle$ provide a resolution of the identity and a POVM.

By explicit evaluation of the matrix elements, one can easily verify the following useful formulas

$$
\left.\int d \lambda B(\lambda) \otimes B^{*}(\lambda)=|\mathbb{1}\rangle\right\rangle\langle\langle\mathbb{1}|,
$$




$$
\left.\left.\int d \lambda B(\lambda) \otimes B^{\dagger}(\lambda)|A\rangle\right\rangle=\left|A^{T}\right\rangle\right\rangle
$$

which are directly equivalent to Eq. (3) of statement 3.

\section{Bell Measurements}

A Bell measurement is a POVM whose elements are projectors on maximally entangled states. Referring to Eqs. (10) and (13) we argue that any POVM of this kind corresponds to a spanning set whose elements are proportional to unitary operators

$$
\Pi(d \lambda)=|\tilde{U}(\lambda)\rangle\rangle\langle\langle\tilde{U}(\lambda)| d \lambda
$$

where $\tilde{U}(\lambda)$ is a basis with $\tilde{U}(\lambda)=\alpha(\lambda) U(\lambda), U(\lambda)$ unitary, and $\alpha(\lambda)$ cnumber.

As proved in Ref. [6], Bell measurements are the only projector valued POVM's capable of teleportation in the case of pure preparation of the shared resource, which turns out to be necessarily in a maximally entangled state.

In the following we give a brief description of this kind of teleportation scheme. The Hilbert space $\mathcal{H}_{1}$ is prepared in an unknown state $\rho^{(1)}$, whereas $\mathcal{H}_{2} \otimes \mathcal{H}_{3}$ is in the maximally entangled state $\left.\frac{1}{\sqrt{N}}|\mathbb{1}\rangle\right\rangle_{23}\left(\mathcal{H}_{1,2,3}\right.$ have the same dimension). Upon performing the measurement described by the POVM (16) on $\mathcal{H}_{1} \otimes \mathcal{H}_{2}$, the (unnormalized) state on $\mathcal{H}_{3}$ conditioned by the outcome $\lambda$ will be

$$
\begin{aligned}
\tilde{\varrho}_{\lambda}^{(3)} & =\operatorname{Tr}_{12}\left[\rho^{(1)} \otimes|\mathbb{1}\rangle\right\rangle_{23}{ }_{23}\left\langle\left\langle\mathbb{1}|| \tilde{U}_{\lambda}\right\rangle\right\rangle_{12}{ }_{12}\left\langle\left\langle\tilde{U}_{\lambda}\right| \otimes \mathbb{1}_{3}\right]= \\
& =\operatorname{Tr}_{12}\left[\rho^{(1)} \otimes|\mathbb{1}\rangle\right\rangle_{23}{ }_{23}\left\langle\langle\mathbb{1}| \tilde{U}_{\lambda}^{(1)} \otimes \mathbb{1}^{(23)} \times\right. \\
& \times|\mathbb{1}\rangle\rangle_{1212}\left\langle\langle\mathbb{1}| \otimes \mathbb{1}^{(3)} \tilde{U}_{\lambda}^{\dagger(1)} \otimes \mathbb{1}^{(23)}\right]= \\
& ={ }_{12}\langle\langle\mathbb{1}|| \mathbb{1}\rangle\rangle_{23} \tilde{U}_{\lambda}^{\dagger(1)} \rho^{(1)} \tilde{U}_{\lambda}^{(1)}{ }_{23}\langle\langle\mathbb{1}|| \mathbb{1}\rangle\rangle_{12}=\tilde{U}_{\lambda}^{\dagger(3)} \rho^{(3)} \tilde{U}_{\lambda}^{(3)} .
\end{aligned}
$$

The normalized state writes

$$
\varrho_{\lambda}=U^{\dagger}(\lambda) \rho U(\lambda)
$$

and the teleportation can be completed upon applying the unitary transformation $U(\lambda)$ on the state (18). If the shared entangled resource is prepared in another maximally entangled state, i.e. $\left.\frac{1}{\sqrt{N}}|V\rangle\right\rangle_{23}$ with $\mathrm{V}$ unitary, it is enough 
to substitute $U(\lambda)$ with $U(\lambda) V^{*}$.

Notice that the product ${ }_{12}\langle\langle\mathbb{1}|| \mathbb{1}\rangle\rangle_{23}$ that appears in Eq. (17) corresponds to the transfer operator $\tau_{31}$ of Ref. [7], which for any vector $|\psi\rangle_{1}$ of $\mathcal{H}_{1}$ satisfies the relation

$$
\tau_{31}|\psi\rangle_{1}=|\psi\rangle_{3}
$$

If the set $\{\tilde{U}(\lambda)\}$ is an orthonormal operator basis (Dirac-like orthonormality relations are allowed in the case of infinite dimensional spaces), it is possible to write the class of Bell observables, i.e. the self-adjoint operators that one has to measure in order to realize the Bell measurement. The Bell observables can be written as follows

$$
\left.O=\int f(\lambda)|\tilde{U}(\lambda)\rangle\right\rangle\langle\langle\tilde{U}(\lambda)| d \lambda
$$

where $f(\lambda)$ must be an injective function (i.e. O is non degenerate) in order to guarantee a univocal correspondence between the read eigenvalue $f(\lambda)$ and the unitary operator $U(\lambda)$ of Eq. (18) that completes the teleportation scheme.

\subsection{The role of group representations}

Unitary irreducible representations (UIR) of groups provide a method to generate a spanning set of unitary operators in the sense of statements (1-4). In fact, if $\left\{U_{g}, g \in \mathbf{G}\right\}$ are the elements of a projective UIR of the group $\mathbf{G}$, from the first Schur's lemma it follows that

$$
\int_{G} d g U_{g} A U_{g}^{\dagger}=\operatorname{Tr}[A] \mathbb{1},
$$

where $d g$ is a (suitably normalized) group invariant measure on $\mathbf{G}$. Recalling Eq. (16), it follows that the POVM

$$
\left.\Pi(d g)=\left|U_{g}\right\rangle\right\rangle\left\langle\left\langle U_{g}\right| d g\right.
$$

describes a Bell measurement.

For example, as noticed in Ref. [7], the N-dimensional UIR of the group $\mathbb{Z}_{N} \times$ $\mathbb{Z}_{N}$ whose elements are

$$
U(m, n)=\sum_{k} e^{2 \pi i k m / N}|k\rangle\langle k \oplus n|
$$


generates the Bell measurement corresponding to the teleportation scheme of Ref. [3].

As an example for the infinite dimensional case, consider the displacement operators of an electromagnetic field mode $a\left(\left[a, a^{\dagger}\right]=1\right)$

$$
D(z)=\exp \left(z a^{\dagger}-z^{*} a\right), \quad z \in \mathbb{C} .
$$

Such operators are the elements of a projective UIR representation of the WeylHeisenberg group $W H$, and generate the Bell measurement corresponding to the Braunstein-Kimble teleportation scheme of Ref. [8].

For $\mathbb{Z}_{N} \times \mathbb{Z}_{N}$ the class of Bell observables defined by Eq. (20) is given by

$$
\begin{aligned}
O & \left.=\sum_{g} f(g)\left|U_{g}\right\rangle\right\rangle\left\langle\left\langle U_{g}\right|=\sum_{g} f(g) U_{g} \otimes \mathbb{1} \sum_{g^{\prime}} U_{g^{\prime}} \otimes U_{g^{\prime}}^{*} U_{g}^{\dagger} \otimes \mathbb{1}=\right. \\
& =\sum_{m, n} \sum_{m^{\prime}, n^{\prime}} f(m, n) e^{\frac{2 \pi i}{N}\left(n m^{\prime}-m n^{\prime}\right)} U\left(m^{\prime}, n^{\prime}\right) \otimes U^{*}\left(m^{\prime}, n^{\prime}\right)= \\
& =\sum_{g} \tilde{f}(g) U_{g}^{(1)} \otimes U_{g}^{(2) *}
\end{aligned}
$$

where we used Eq. (14) along with the relation

$$
U(m, n) U\left(m^{\prime}, n^{\prime}\right) U^{\dagger}(m, n)=e^{\frac{2 \pi i}{N}\left(n m^{\prime}-m n^{\prime}\right)} U\left(m^{\prime}, n^{\prime}\right)
$$

and we introduced the Fourier transform $\tilde{f}$ over the group

$$
\tilde{f}(m, n)=\sum_{m^{\prime}, n^{\prime}} e^{\frac{2 \pi i}{N}\left(n m^{\prime}-m n^{\prime}\right)} f\left(m^{\prime}, n^{\prime}\right) .
$$

By applying Eq. (14), the analogous relation for $W H$ reads as follows

$$
\begin{aligned}
O & \left.=\int_{\mathbb{C}} d^{2} z f(z)|D(z)\rangle\right\rangle\langle\langle D(z)|= \\
& =\int_{\mathbb{C}} d^{2} z f(z) D(z) \otimes \mathbb{1} \int_{\mathbb{C}} \frac{d^{2} \alpha}{\pi} D(\alpha) \otimes D\left(\alpha^{*}\right) D(z)^{\dagger} \otimes \mathbb{1}= \\
& =\int_{\mathbb{C}} d^{2} \alpha \int_{\mathbb{C}} \frac{d^{2} z}{\pi} f(z) e^{\alpha z^{*}-\alpha^{*} z} D(\alpha) \otimes D\left(\alpha^{*}\right)= \\
& =\int_{\mathbb{C}} d^{2} \alpha \tilde{f}(\alpha) D(\alpha) \otimes D\left(\alpha^{*}\right) .
\end{aligned}
$$


However, in this case, one can derive a more explicit expression for the Bell observables. In fact, from Eq. (14) one has

$$
\begin{aligned}
|\mathbb{1}\rangle\rangle_{1212}\langle\langle\mathbb{1}| & =\int_{\mathbb{C}} \frac{d^{2} \beta}{\pi} D_{1}(\beta) \otimes D_{2}\left(\beta^{*}\right)= \\
& =\int_{\mathbb{C}} \frac{d^{2} \beta}{\pi} \exp \left[\left(\beta a_{1}^{\dagger}-\beta^{*} a_{1}\right)+\left(\beta^{*} a_{2}^{\dagger}-\beta a_{2}\right)\right]= \\
& =\int_{\mathbb{C}} \frac{d^{2} \beta}{\pi} \exp \left[\beta Z_{12}^{\dagger}-\beta^{*} Z_{12}\right] \doteq \pi \delta^{(2)}\left(Z_{12}\right),
\end{aligned}
$$

with $Z_{12}=a_{1}-a_{2}^{\dagger}$. Using the relation $D_{a}(z) a D_{a}^{\dagger}(z)=a-z$, one obtains

$$
\left.\frac{1}{\pi}|D(z)\rangle\right\rangle_{1212}\left\langle\langle D(z)|=\delta^{(2)}\left(Z_{12}-z\right)\right.
$$

and finally

$$
\left.O=\int_{\mathbb{C}} d^{2} z f(z) \frac{1}{\pi}|D(z)\rangle\right\rangle_{1212}\left\langle\langle D(z)|=f\left(Z_{12}\right) .\right.
$$

Hence, in order to realize the Bell measurement generated by $W H$, we have to measure an injective function of the operator $Z_{12}$, or simply $Z_{12}$ itself. This measurement can be easily performed by unconventional heterodyne detection (cfr. Ref. [9]).

\section{Robustness of "pure" teleportation}

"Pure" teleportation schemes rely on projector valued POVM's and pure preparations for the shared resource. As proved in Ref. [6], this kind of teleportation works properly if and only if the elements of the POVM are proportional to projectors on maximally entangled states and the resource itself is maximally entangled. However, for practical purposes, one is interested in the evaluation of the robustness of this kind of schemes to non ideality.

Looking at Eq. (17), it is evident that the state on $\mathcal{H}_{3}$ conditioned by the measurement is a continuous function of the shared resource preparation and of the element of the POVM related to the outcome. Since the teleported state is again a continuous function of this conditioned state and of the "adjusting" unitary transformation, we conclude that teleportation is robust to non ideal entanglement preparation, non ideal measurement, and non ideal adjusting transformation. 
Let's suppose that before the measurement the maximally entangled resource evolves according to a trace preserving $\mathrm{CP}$ map $\mathcal{E}$ owing to some kind of noise. In the following, we will simply evaluate the state on $\mathcal{H}_{3}$ after the measurement in presence of such a noise.

By means of the Kraus's decomposition [10] of $\mathcal{E}$, the noisy state of $\mathcal{H}_{2} \otimes \mathcal{H}_{3}$ can be written as

$$
\left.\rho_{23}=\mathcal{E}\left(\frac{1}{N}|\mathbb{1}\rangle\right\rangle_{2323}\langle\langle\mathbb{1}|)=\sum_{\mu} A_{\mu}^{(23)} \frac{1}{N}|\mathbb{1}\rangle\right\rangle_{23}{ }_{23}\left\langle\langle\mathbb{1}| A_{\mu}^{(23) \dagger}\right.
$$

where $A_{\mu}$ are operators on $\mathcal{H}_{2} \otimes \mathcal{H}_{3}$ satisfying $\sum_{\mu} A_{\mu}^{\dagger} A_{\mu}=\mathbb{1}$.

For any (generally non local) operator $\mathrm{A}$ acting on $\mathcal{H} \otimes \mathcal{H}$ one has

$$
\left.\left.A|\mathbb{1}\rangle\rangle=\left|\hat{A}^{T}\right\rangle\right\rangle=\mathbb{1} \otimes \hat{A}|\mathbb{1}\rangle\right\rangle
$$

where $(\hat{A})_{i, j}=\sum_{l}\langle i|\langle j|A| l\rangle| l\rangle$. Therefore it is possible to write

$$
\begin{aligned}
\mathcal{E}\left(\frac{1}{N}|\mathbb{1}\rangle\right\rangle_{2323}\langle\langle\mathbb{1}|) & \left.=\sum_{\mu} A_{\mu}^{(23)} \frac{1}{N}|\mathbb{1}\rangle\right\rangle_{2323}\left\langle\langle\mathbb{1}| A_{\mu}^{(23) \dagger}=\right. \\
& \left.=\sum_{\mu} \mathbb{1}_{2} \otimes \hat{A}_{\mu}^{(3)} \frac{1}{N}|\mathbb{1}\rangle\right\rangle_{2323}\left\langle\langle\mathbb{1}| \mathbb{1}_{2} \otimes \hat{A}_{\mu}^{(3) \dagger}=\right. \\
& =\mathcal{I}^{(2)} \otimes \hat{\mathcal{E}}^{(3)}\left(\frac{1}{N}|\mathbb{1}\rangle\right\rangle_{2323}\langle\langle\mathbb{1}|)
\end{aligned}
$$

where $\hat{\mathcal{E}}$ is the map whose Kraus's decomposition is given by the operators $\hat{A}_{\mu}$. This last equation shows how the action of any CP map on a maximally entangled state of a bipartite system can be written as the result of the application of a local CP map.

Recalling Eq. (17), it results that the local CP map $\hat{\mathcal{E}}^{(3)}$, which describes noise, commutes with all other maps and with the partial trace, so that the unnormalized conditioned state after the measurement, in presence of such a noise, can be simply written as

$$
\tilde{\varrho}_{\lambda}^{(3)}=\hat{\mathcal{E}}^{(3)}\left(U(\lambda)^{\dagger} \rho U(\lambda)\right)
$$

Now, we will restrict our attention to qubit teleportation with non ideal resource preparation $|S\rangle\rangle_{23}$, it is possible to give an explicit expression for the minimum fidelity achieved by teleportation on pure states.

If $|\psi\rangle$ is the original state, apart from a normalization factor, the teleported state will be

$$
\left|\psi_{\lambda}\right\rangle=U_{\lambda} S^{T} U_{\lambda}^{\dagger}|\psi\rangle
$$


where $U_{\lambda}$ is the unitary operator related to the outcome $\lambda$.

The minimum fidelity achieved by teleportation can be written as follows

$$
F_{\text {min }}=\min _{|\psi\rangle \in \mathcal{H}} \frac{\left|\left\langle\psi\left|U_{\lambda} S^{T} U_{\lambda}^{\dagger}\right| \psi\right\rangle\right|^{2}}{\left\langle\psi\left|U_{\lambda} S^{*} U_{\lambda}^{\dagger} U_{\lambda} S^{T} U_{\lambda}^{\dagger}\right| \psi\right\rangle}=\min _{|\psi\rangle \in \mathcal{H}} \frac{\left|\left\langle\psi\left|S^{T}\right| \psi\right\rangle\right|^{2}}{\left\langle\psi\left|S^{*} S^{T}\right| \psi\right\rangle} .
$$

Using a basis of $\mathcal{H}_{2} \otimes \mathcal{H}_{3}$ for which $\left.|S\rangle\right\rangle_{23}$ is in the Schmidt-form, i.e. diagonal and positive, and noticing that $F_{\min }$ is independent of the normalization of $S$, we can choose $S$ to be

$$
S_{\epsilon}=(1+\epsilon)|0\rangle\langle 0|+(1-\epsilon)| 1\rangle\langle 1|
$$

The minimization can performed only on states $|\psi(x)\rangle$ of the form

$$
|\psi(x)\rangle=\cos x|0\rangle+\sin x|1\rangle, \quad x \in[0,2 \pi)
$$

because any phase would be irrelevant. Substituting Eqs. (38) and (39) in Eq. (37) and minimizing respect to $x$, one obtains

$$
F_{\text {min }}=1-\epsilon^{2}
$$

With some little algebra, Eq. (40) can be cast in a compact form independent of basis and normalization as follows

$$
F_{\text {min }}=4 \frac{\operatorname{det}(\tilde{S})}{\operatorname{Tr}^{2}[\tilde{S}]}, \quad \text { where } \quad \tilde{S}=\sqrt{S^{\dagger} S}
$$

\section{Conclusions}

We studied the problem of characterizing Bell measurements, as maximally entangled POVM's for measurements on composite systems. We introduced operator-completeness relations and a simple matrix approach to deal with bipartite systems. These allow us to write the most general Bell-like POVM in a compact form. The role of spanning sets of unitary operators has been emphasized, with attention to unitary irreducible representations of groups. Bell observables related to Bell POVM's have been explicitly derived. As direct application of the matrix formalism, we evaluated the robustness of teleportation to non maximality of the shared entangled resource and to non ideality

of the measurement. 


\section{References}

[1] Introduction to Quantum Computation and Information, H-K Lo, S. Popescu, and T. Spiller, Eds., World Scientific, Singapore, 1998.

[2] D. Boumeester, J.-W. Pan, K. Mattle, M. Eibl, H. Weinfurter and A. Zeilinger, Nature (London) 390, 575 (1997); D. Boschi, S. Branca, F. De Martini, L. Hardy and S. Popescu, Phys. Rev. Lett. 80, 1121 (1998); A. Furasawa, J.L. Sørensen, S.L. Braunstein, C.A. Fuchs, H.J. Kimble and E.S. Polzik, Science 282, 706 (1998); A. Muller, H. Zbinden, and N. Gisin, Europhys. Lett. 33, 35 (1996); J. I. Cirac and P. Zoller, Phys. Rev. Lett. 74, 4091 (1995).

[3] C. H. Bennett, G. Brassard, C. Crepeau, R. Jozsa, A. Peres, and W. K. Wooters, Phys. Rev. Lett. 70, 1895 (1993).

[4] A. Royer, Phys. Rev. A 43, 44 (1991).

[5] A. Peres, Quantum Theory: Concepts and Methods (Kluwer Academic, Dordrecht, 1993).

[6] M. A. Nielsen and C. Caves, Phys. Rev. A 55, 2547 (1997).

[7] S. L. Braunstein, G. M. D'Ariano, G. J. Milburn, and M. F. Sacchi, Phys. Rev. Lett. 84, 3486 (2000).

[8] S. L. Braunstein and H. J. Kimble, Phys. Rev. Lett. 80, 869 (1998).

[9] G. M. D'Ariano and M. F. Sacchi, Phys. Rev. A 52, R4309 (1995); G. M. D'Ariano and M. F. Sacchi, Mod. Phys. Lett. B. 11, 1263 (1997).

[10] K. Kraus, States, effects, and Operations, (Springer-Verlag, Berlin, 1983). 